\title{
Algorithm for Reducing the Effect of Network Delay of Sensor Data in Network-Based AC Motor Drives
}

\author{
Tae-Won Chun ${ }^{\dagger}$, Jung-Ryol Ahn*, Hong-Hee Lee**, Heung-Geun Kim***, and Eui-Cheol Nho \\ $\dagger * *$ Dept. of Electrical Eng., University of Ulsan, Ulsan, Korea \\ * Dept. of Electrical and Electronics Eng., Donghee Industrial Co. Ltd., Ulsan, Korea \\ *** Dept. of Electrical Eng., Kyungpook National University, Daegu, Korea \\ ¥Dept. of Electrical Eng., Pukyong National University, Busan, Korea
}

\begin{abstract}
Network-based controls for ac motor drive systems are becoming increasingly important. In this paper, an ac motor control system is implemented by a motor control module and three sensor modules such as a voltage sensor module, a current sensor module, and an encoder module. There will inevitably be network time delays from the sensor modules to the motor control system, which often degrades and even destabilizes the motor drive system. As a result, it becomes very difficult to estimate the network delayed ac sensor data. An algorithm to reduce the effects of network time delays on sensor data is proposed, using both a synchronization signal and a simple method for estimating the sensor data. The algorithm is applied to a vector controlled induction motor drive system, and the performance of the proposed algorithm is verified with experiments.
\end{abstract}

Key Words: AC motor, Control area network (CAN), Network delay, Sensor modules, Vector control

\section{INTRODUCTION}

Flexibility of operation is required in modern industrial systems. This requirement is motivated by the need to reduce the cost of setup, configuration change, and maintenance and it extends to all system levels, including the field level in process industries and the cell and plant levels in manufacturing industries, where fieldbus networks are commonly found. Networkbased control for an ac motor drive system, which has wide use as an actuator in the industrial automation systems, is becoming increasingly important. The supervisory control method for multi-motors via a fieldbus network has been suggested [1], [2]. The host PC sends reference commands to each motor drive system and monitors the operating conditions for the multi-motors throughout the network.

One recent advance in sensor technology is sensor modulization including network function, self-diagnosis as well as data measurement, which offers greater design flexibility, robustness, and superior functionality [3]. In this paper, an ac motor control system is implemented as four modules: a

Manuscript received Aug. 2, 2010; revised Feb. 18, 2011

Recommended for publication by Associate Editor Kyeong-Hwa Kim.

$\dagger$ Corresponding Author: twchun@mail.ulsan.ac.kr

Tel: +81-52-259-2188, Fax: +81-52-259-1686, University of Ulsan

* Dept. of Electrical and Electronics Eng., Donghee Industrial Co. Ltd., Korea

** Dept. of Electrical Eng., University of Ulsan, Korea

*** Dept. of Electrical Eng., Kyungpook National University, Korea

$\ddagger$ Dept. of Electrical Eng., Pukyong National University, Korea voltage sensor module, a current sensor module, an encoder module and a motor control module. The ac motor is controlled by sensor data received from the three sensor modules through the network. As the ac motor control is closed over a fieldbus, there will inevitably be a random time delay from the sensors to the control system. This network induced delay can degrade the performance of the control system and can even destabilize the motor drive system.

An IEEE 1451-based smart modules including a motor control module, an encoder module, and a monitor module have been developed [4]. Some papers focused on analyzing the stability and designing the controller of a networked control system with random or time-varying time delays [5]-[9]. With the development of the internet, more internet-based control applications such as telerobotics and teleoperations will emerge in the near future [10]. However, for real-time internetbased control systems, the effects of random time transmission delays and data drops caused by a router cannot be ignored. The worst case communication delay of a switched Ethernet with multiple levels has been derived, and the efficiency of the switched Ethernet has been investigated by examining the performance of the network control systems [11], [12]. A method of using a gain schedule middleware (GSW) on a proportional-integral (PI) controller for dc motor speed control over network delays has been introduced [13]. Networked predictive control methods using a model-based predictive control algorithm or by selecting the control signal according 
to the system output rather than time delay measurements have been proposed [14], [15].

Transmission scheduling algorithms have been proposed for reducing the effects of network induced delays. The most natural scheduling methods are a fixed priority type or a dynamic priority type of scheduling by changing the message priority dynamically [16]. However, these methods can not be applied to the system suggested in this paper, because the ac current and voltage sensor modules among the three sensor modules have the same high priority level. A predictive control to compensate for delayed sensing data and a scheduling codesign approach are proposed to deal with the controller and the scheduler design for networked control systems which are connected to a shared communication network [17]. A time delay compensator on the actuator side is designed to compensate for network induced delays, using delayed sensing data and previous information. However, for online implementation, calculating the control predictions is a great burden for the controller.

In this paper, an algorithm to reduce the effects of network time delays on sensor data is proposed, when all sensor data such as the three phase voltages and currents, the speed and/or the position are received through a network for controlling ac motors. The network delay time from the sensor modules to the control module is kept constant using a synchronization signal and timers, and the sensor data is estimated considering the network delay time. The algorithm is applied to a vector controlled induction motor drive system.

\section{NETWORK STRUCTURE}

The network hierarchy in manufacturing systems can be classified according to the device level, the cell level, and the plant level. A fieldbus is chosen for communicating real-time data between the an actuator and its sensors at the device level, while the Ethernet is used for communicating non real-time data among actuators and PLCs at the cell level, or among industrial computers and database systems at the plant level.

Fig. 1 shows the network structure for an ac motor drive system at the device level. It consists of a current sensor module, a voltage sensor module, an encoder module, and a motor control module. At the three sensor modules, the threephase ac currents, the three-phase ac voltages, the speed and/or the position of the rotor are measured and transmitted to the motor control module through the network, respectively. At the motor control module, the speed and/or the position of the ac motor driven by a PWM inverter is controlled using the sensor data received from the three sensor modules. A control area network (CAN) is used for real-time communication between the sensor modules and the control module. The CAN was originally developed to support low cost simple automotive applications. However, due to its performances and economic feasibility, it is now becoming very popular as a fieldbus for automated manufacturing [18], [19].

Fig. 2 shows the configurations of the sensor modules. The sensor modules use a 16-bit DSP TMS320LF2406A with a $30 \mathrm{MHz}$ clock frequency, which contains flash memory, an 8channel 10-bit A/D converter, 16-bit timers/counters, a QEP,

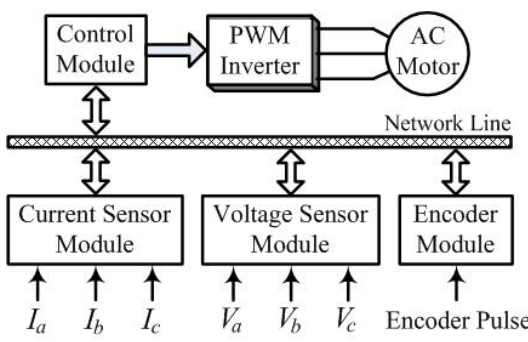

Fig. 1. Network structure.

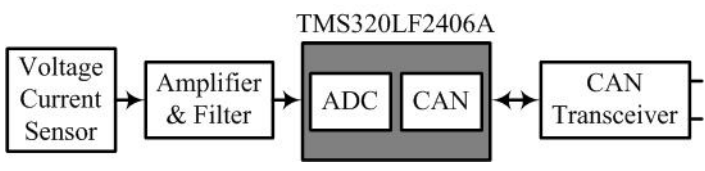

(a)

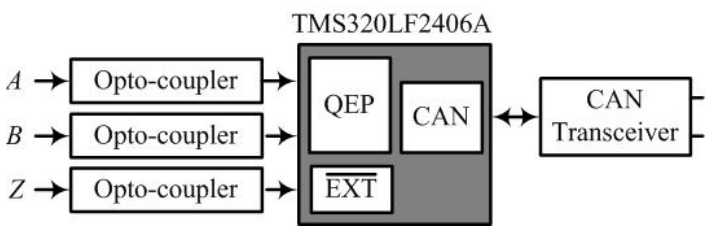

(b)

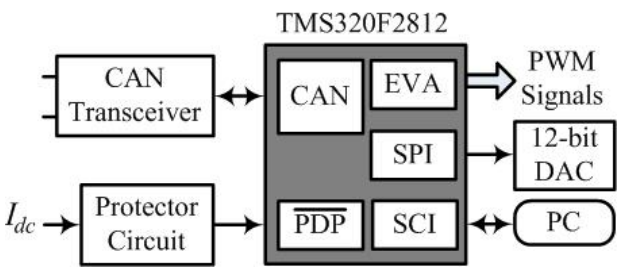

(c)

Fig. 2. Configuration of modules. (a) current and voltage sensor modules. (b) encoder module. (c) motor control module.

a CAN controller, etc. As shown in Fig. 2(a), the functions for sensing, amplifying, filtering and converting the analog sensor data into digital values using an A/D converter, and transmitting the sensor data through the CAN transceiver are implemented in the current and voltage sensor modules. At the encoder module shown in Fig. 2(b), after isolating the output signals of the encoder with photo couplers, the speed and position can be calculated by counting the output pulses of the encoder. They are then transmitted through the CAN.

Fig. 2(c) shows the motor control module, where the sensor data are received from the sensor modules through the network. For the stator flux oriented vector control, the d- and qaxis stator fluxes can be calculated from the motor terminal voltage and current as follows [20]:

$$
\begin{aligned}
& \psi_{d s}^{s}=\int\left(V_{d s}^{s}-R_{s} i_{d s}^{s}\right) d t \\
& \psi_{q s}^{s}=\int\left(V_{q s}^{s}-R_{s} i_{q s}^{s}\right) d t .
\end{aligned}
$$

The vector controlled induction motor drive system is implemented by a high performance 32-bit DSP, TMS320F2812 with a $150 \mathrm{MHz}$ clock frequency. Six PWM signals with dead time are generated, and they are blocked when over-current occurs. A 12-bit 4-channel D/A converter is used to investigate some waveforms through an oscilloscope. The baudrate of the 


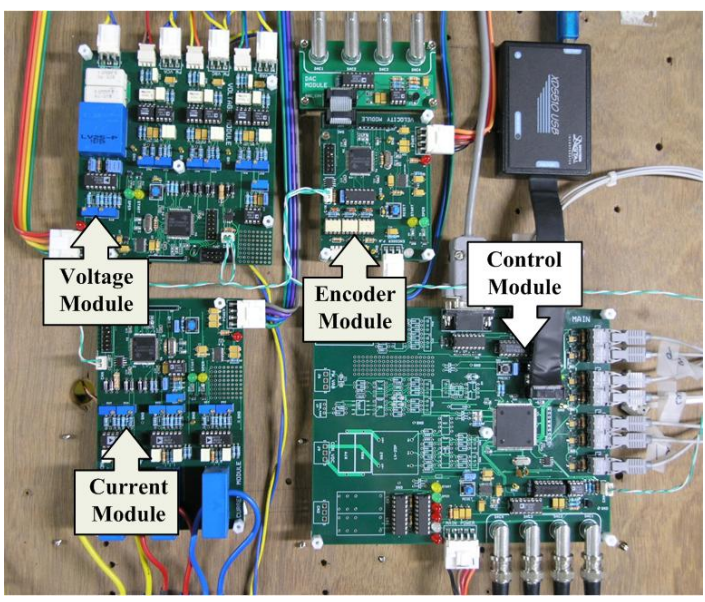

Fig. 3. Photograph of the modules.

CAN is 2Mbps, and a TI SN657VD23 is used as the CAN transceiver.

Fig. 3 shows a photograph of the control and the three sensor modules.

\section{METhod OF DATA COMMUNiCATION}

\section{A. Method for transmitting sensor data}

Since the three sensor modules are independently operated, the sensor modules transmit their data asynchronously to the network. Therefore, the time delay from the sensors to a controller is randomly varied, and the delay time may be larger than a sampling period. An unexpected large delay of sensor data, particularly ac voltages and currents may deteriorate the performance of a system or make it unstable.

In this paper, the sensor data measured by the three sensor modules is transmitted synchronously to the motor control module in order to keep the network induced delay time constant. Fig. 4 shows the method used to communicate the sensor data, using the synchronization signal generated by the control module and timers for measuring the time to transmit the sensor data from each of the sensor modules.

At the control module, the synchronization signal is sent simultaneously to the three sensor modules following every three sequences of the sampling period. Since a CAN identifier is made up of a sequence of 11 bits and the size of the data is 8 bits, the size of the packet frame of the synchronization signal is only 52 bits. The network induced delay time from the control module to the sensor modules $\tau_{d 1}$ is $26 \mu \mathrm{sec}$ if the baudrate of the CAN is $2 \mathrm{Mbps}$. When the sensor modules receive a signal from the control module after a delay time $\tau_{d 1}$, the timers embedded on the DSP in the each of the sensor modules are cleared at the same time. The timers are used to measure the time required to transmit the sensor data to a control module. The current sensor module transmits three-phase ac currents to the control module, when the synchronization signal is received. When the timer reaches the sampling time, the voltage sensor module transmits threephase ac voltages to the control module. During the next sampling period, the encode module transmits both the speed and position to the control module.

Fig. 5 shows the operation of the timers of the three sensor modules in response to the synchronization signal when the

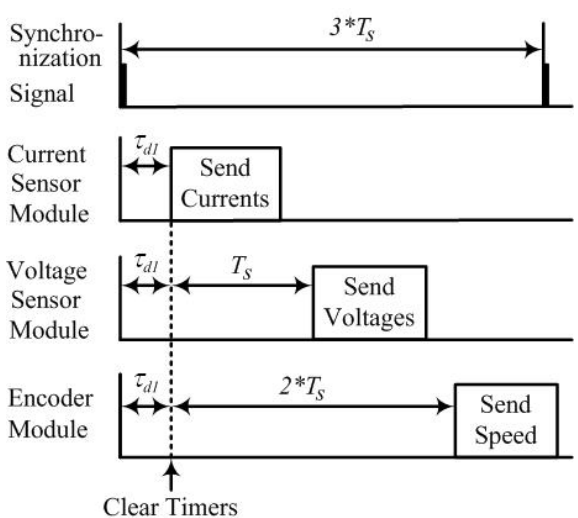

Fig. 4. Timing for the synchronization signal and transmission of the sensor data.

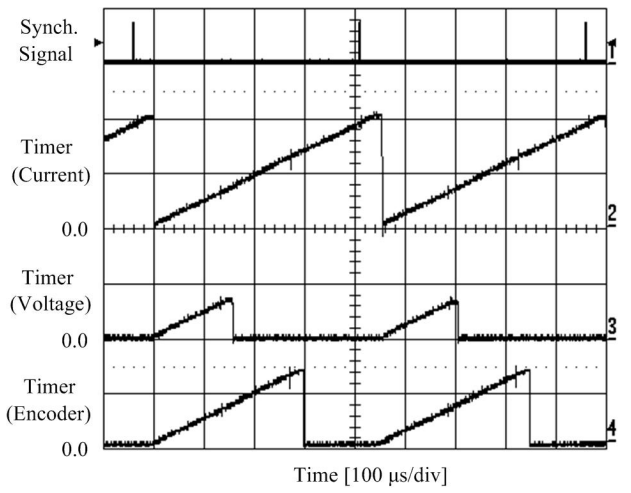

Fig. 5. Synchronization signal and timers.

sampling period $T_{S}$ is $150 \mu \mathrm{sec}$. When the sensor modules receive the synchronization signal, all of the timers are cleared. Whenever the timer of each of the sensor modules matches $0 \mu \mathrm{sec}, 150 \mu \mathrm{sec}$ or $300 \mu \mathrm{sec}$, the sensor modules transmit their data.

\section{B. Data communication of the motor control module}

Fig. 6 shows the timing for receiving the sensor data at the control module, where it has three tasks: a current reception task, a voltage reception task, and an encoder reception task. Upon the receipt of a synchronization signal, the three sensor modules transmit the measured data sequentially to the control module during each sampling period. The CAN receive interrupt is generated after a delay time $\tau_{d 2}$, which is about $46 \mu$ sec. Since only one measured sensor data among the three sensor modules can be received in one sampling period during three sampling periods, the sensor data for the next two sampling periods should be estimated from the received sensor data. Thus, one measured sensor data and two estimated sensor data are available for motor control.

At the current reception task, three phase currents are received, and then the three-phase currents for the next two sampling periods should be estimated. The currents received from the current sensor module, and the voltages and speed (position) estimated from a previous sampling period are used for controlling the motor. At the voltage reception task, after receiving the three phase voltages, the estimated three phase voltages are calculated. The motor control is then executed with the received voltages, and the estimated currents and 


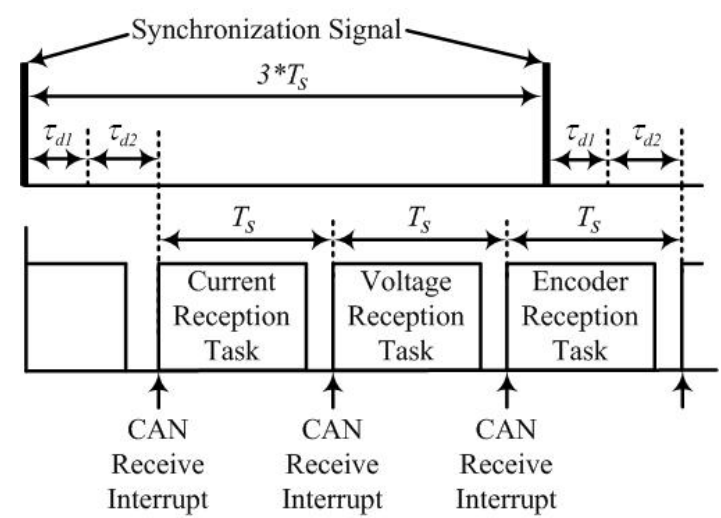

Fig. 6. Timing for receiving the sensor data.

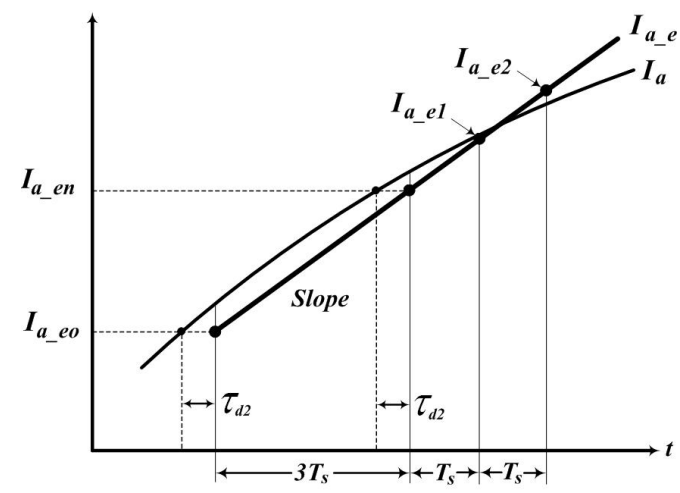

Fig. 7. Method for estimating the sensor data.

speed (position). At the encoder reception task, after receiving and estimating both the speed and position, the speed (position) control of the motor is executed with the received speed (position), and the estimated three phase currents and voltages.

\section{Estimation of SEnsor Data}

Among the three sensor modules, one sensor module transmits its measured data to the control module in one sampling period. Since the control module can receive only one sensor data in one sampling period, the sensor data for controlling the motor in the next two sampling periods should be estimated from the received sensor data.

Fig. 7 shows the method for estimating the a-phase current among the sensor data. Current data can be received every three sampling periods, $3 T_{s}$, and the currents received in the previous step and at the current step are represented by $I_{a_{-} e o}$ and $I_{a \_n}$, respectively. It can be seen that there is an error between the motor current and the received current due to the network induced delay $\tau_{d 2}$.

The slope of the a-phase currents received in both steps is expressed as:

$$
\text { Slope }=\frac{I_{a_{-} e o}-I_{a_{-} e n}}{3 T_{S}}
$$

Using the slope, the estimated currents in the first sampling period and in the second sampling period can be calculated as shown by the following equations, respectively.

$$
\begin{gathered}
I_{a \_e 1}=I_{a \_e n}+T_{S} \times \text { Slope } \\
I_{a \_e 2}=I_{a \_e n}+2 T_{S} \times \text { Slope }
\end{gathered}
$$

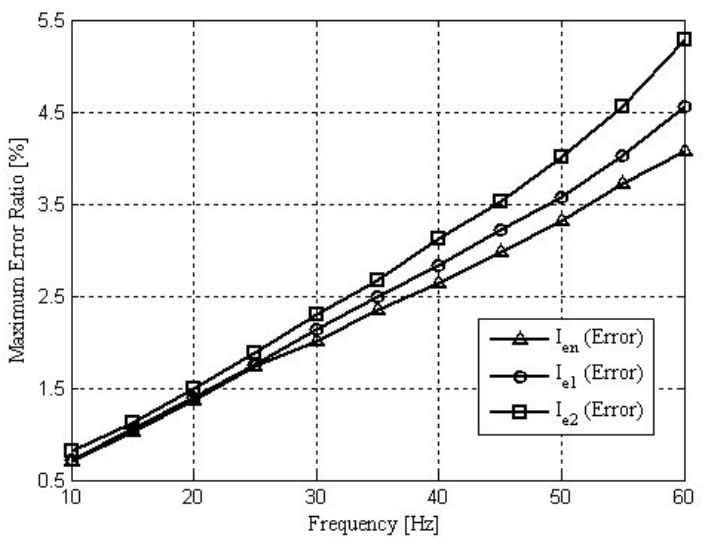

Fig. 8. Errors of the received and estimated currents.

There should be the current errors between the actual current and received/estimated currents, and the errors are varied in terms of both the phase and frequency of the ac current. The ratio of the maximum errors in one received current and two estimated currents to the peak current during one period with a variation of the inverter frequency are plotted in Fig. 8. The three current errors are increased with the inverter frequency, and they may be less than the current error caused in one sampling period.

\section{EXPERIMENTAL RESULTS}

Fig. 9(a) shows the experimental results for the a-phase motor current, the current received from a current module through the network, and the current error, when three sensor modules transmit their data randomly to the motor control module. The maximum current error between the motor current and the received current is around $17 \%$ of the peak motor current, and the received current lags the motor current by about $9^{\circ}$. From Fig. 9(b), it can be seen that sometimes the current can not be received during two or three sampling periods due to the amount of network traffic.

Fig. 10 shows the experimental results, when the algorithm proposed in this paper is applied. The maximum current error can be reduced to about $3 \%$ of the peak motor current, and the phase lag of the estimated current to the real current is within the boundary of one sampling period as shown in Fig. 10(b). It can be seen that the ratio of the maximum current error, as shown in Fig. 8, is about 2.8\%, when the inverter frequency is $33 \mathrm{~Hz}$

Fig. 11 shows the waveforms of the TX signals of the CAN in a control module and three sensor modules. After transmitting the synchronization signal, the sensor data are transmitted in every sampling period, $150 \mu$ sec through the TX line.

Fig. 12 shows the experimental results for the transient responses of the motor speed and the d-and q-axis currents in a vector controlled induction motor drive system using sensor modules, when the motor starts up to 500rpm and then when it accelerates from 500rpm to 1000rpm with and without the proposed estimation method, respectively. The motor speed reaches its reference speed, and the d-axis current is controlled to the rated magnetizing current. The q-axis current is nearly equal to zero in the steady state condition, 


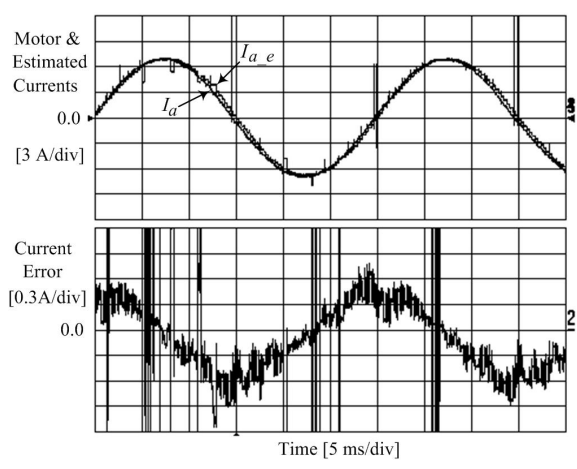

(a)

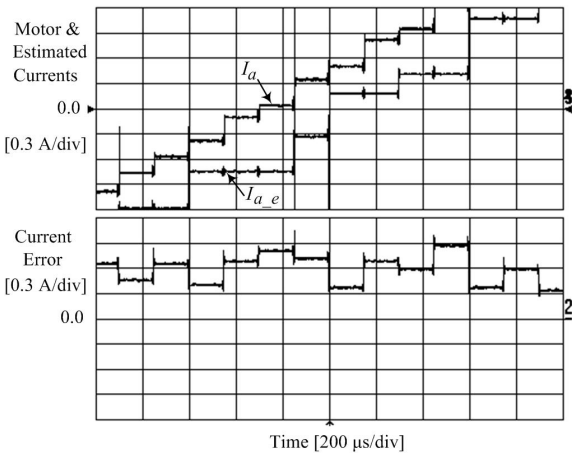

(b)

Fig. 9. Experimental results for currents when the sensor modules randomly transmit data. (a) currents. (b) magnified figures of the positive zero-crossing of the motor current in (a)

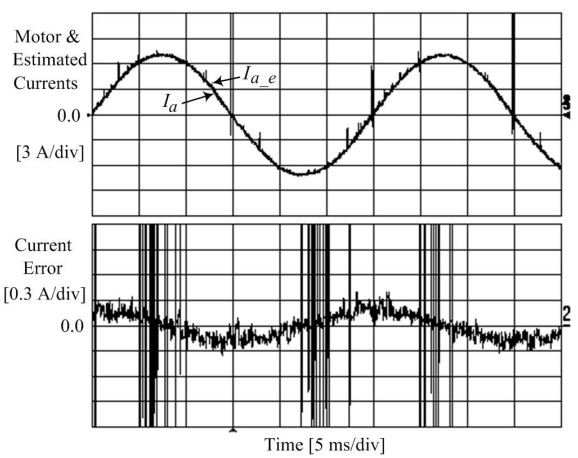

(a)

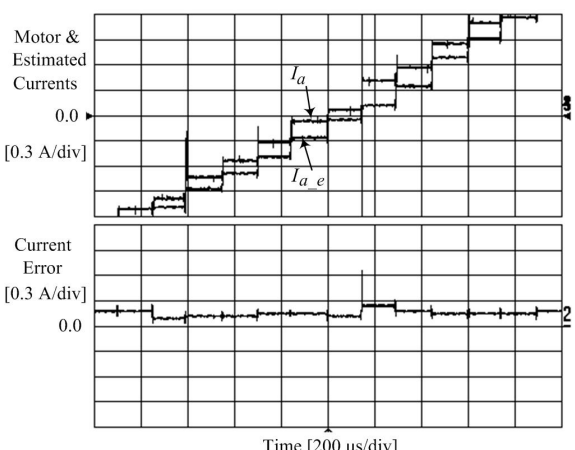

(b)

Fig. 10. Experimental results for currents when the estimation method is applied. (a) currents. (b) magnified figures of the positive zero-crossing of motor current in (a).

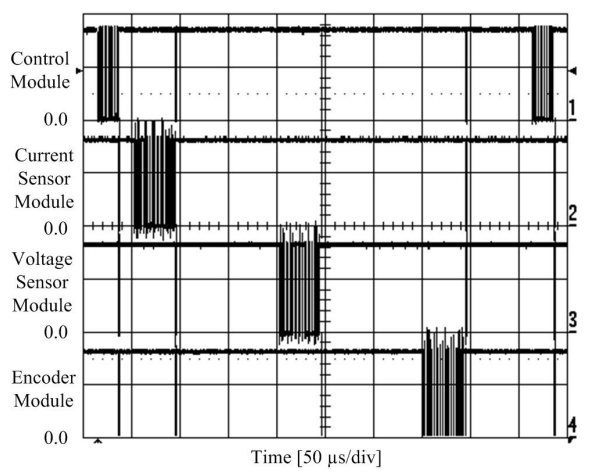

Fig. 11. TX signals of CAN in the four modules.

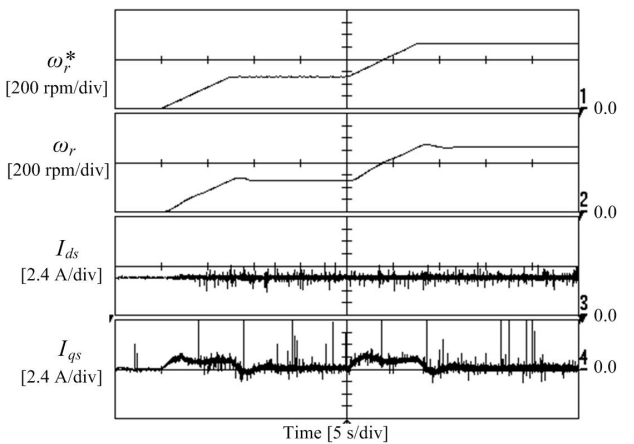

(a)

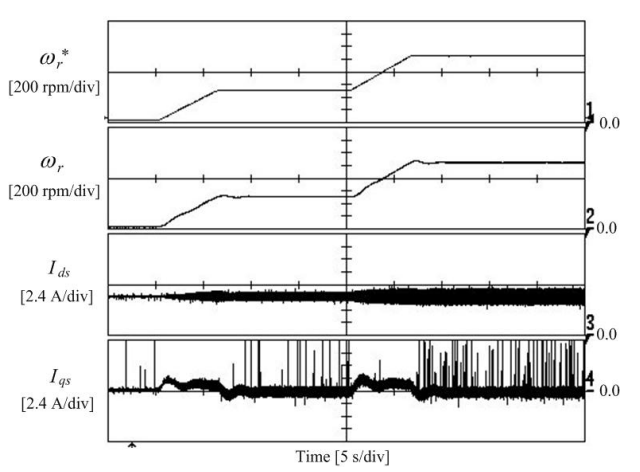

(b)

Fig. 12. Transient responses at the start-up. (a) with the estimation method (b) without the estimation method.

because a flywheel with a high moment of inertia is used as a load for the induction motor. When the sensor data is randomly transmitted, the ripple components of the d- and q-axis currents are increased as shown in Fig. 12(b).

Fig. 13 shows the experimental result with the proposed estimation method, when the reference speed is changed more rapidly from $500 \mathrm{rpm}$ to $1000 \mathrm{rpm}$, and then back to $500 \mathrm{rpm}$.

\section{CONCLUSIONS}

In this paper, an algorithm was proposed to reduce the effects of network time delays on sensor data, when three phase voltages and currents, and the speed and/or position are received through a CAN for driving an induction motor. When the three sensor modules transmit their data randomly to the control module, the maximum data error between the actual sensor data and the sensor data received from the network was around $17 \%$. When the method of transmitting sensor data synchronously to a control module and estimating the 


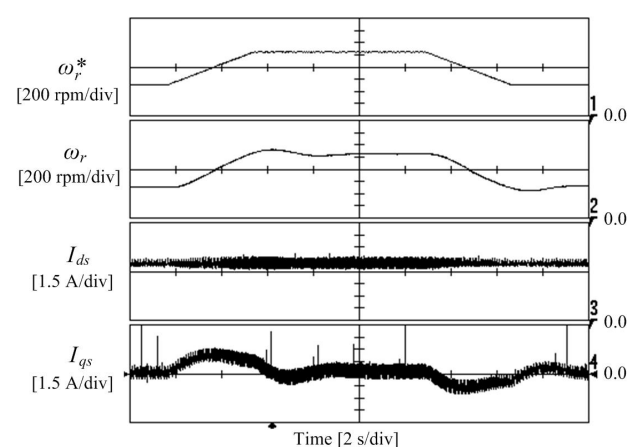

Fig. 13. Transient responses when the reference speed is changed.

sensor data is applied, the maximum sensor data error can be reduced to $3 \%$. Furthermore, the phase lag of the estimated sensor data to the actual sensor data was within the boundary of a sampling period by a simple slope method. The algorithm is applied to a vector controlled induction machine, and the transient responses for the motor speed and currents are good.

\section{REFERENCES}

[1] H. H. Lee, E. H. Jung, and K. S. Lim, "Robust induction motor control on network-based control systems," in Annual Conference of IEEEIndustrial Electronics Society, pp. 72-76, 2003.

[2] J. Bosteels, "Coordinated multi-axis motion control via CANopen," CAN Newsletter, Feb. 2002.

[3] C. Y. Chong and S. P. Kumar, "Sensor Networks: Evolution, Opportunities, and Challenges," in Proceedings of IEEE, Vol. 92, No. 8, pp. 1247-1256, 2003.

[4] K. C. Lee, H. H. Kim, S. Lee, and H. H. Lee, "IEEE- 1451-based smart module for in-vehicle networking systems for intelligent vehicles," IEEE Trans. Ind. Electron., Vol. 51, No. 6, pp. 1150-1157, Dec. 2004.

[5] M. Y. Chow and Y. Tipsuwan, "Network-based control systems: A tutorial," in Annual Conference of IEEE-Industrial Electronics Society, pp.1593-1602, 2001.

[6] J. K .Yook, D. M. Tilbury, and N. R. Soparkar, "A design methodology for distributed control systems to optimize performance in the presence of time delay," Int. J. Contr., Vol. 74, No.1, pp.58-76, Jan. 2001.

[7] J. Nilsson, B. Bernhardsson, and B. Wittenmark, "Stochastic analysis and control of real-time systems with random time delay," Automatica, Vol. 34, No. 1, pp. 57-64, 1998.

[8] M. Y. Chow and Y. Tipsuwan, "Gain adaptation of networked DC motor controllers based on QoS variation," IEEE Trans. Ind. Electron., Vol. 50, No. 5, pp. 936-943, Oct. 2003.

[9] G. P. Liu, Y. Xia, J. Chen, D. Rees, and W. Hu, "Networked predictive control of systems with random network delays in both forward and feedback channel," IEEE Trans. Ind. Electron., Vol. 54, No. 3, pp.936943, Jun. 2007.

[10] K. B. Sim, K. S. Byun, and F. Harashima, "Internet-based teleoperation of an intelligent robot with optimal two-layer fuzzy controller," IEEE Trans. Ind. Electron., Vol. 53, No. 4, pp. 1362-1372, Aug. 2006.

[11] K. C. Lee, S. Lee, and M. H. Lee, "Remote fuzzy logic control of network control system via Profibus-DP," IEEE Trans. Ind. Electron., Vol.50, No.4, pp.784-792, Aug. 2003.

[12] K. C. Lee, S. Lee, and M. H. Lee, "Worst case communication delay of real-time industrial switched Ethernet with multiple levels," IEEE Trans. Ind. Electron., Vol.53, No.5, pp.1669-1676, Oct. 2006.

[13] Y. Tipsuwan and M. Y. Chow, "Gain scheduler middleware: A methodology to enable existing controllers for networked control and teleoperation-Part I: Networked control," IEEE Trans. on Ind. Electron., Vol.51, No.6, pp.1218-1227, Dec. 2004.

[14] G. P. Liu, J. X. Mu, D. Rees, and S. C. Chai, "Design and stability analysis of networked control systems with random communication time delay using the modified MPC," Int. J. Control, Vol.59, No.4, pp288297, Apr. 2006.

[15] W. S. Hu and G. P. Liu, "Event-driven networked predictive control," IEEE Trans. on Ind. Electron., Vol. 54, No. 3, pp.1603-1613, Jun. 2007.

[16] T. Nolte, M. Nolin, and H. A. Hansson, "Real-time server-based communication with CAN," IEEE Trans. Industrial Informatics, Vol. 1, No. 3, pp. 192-201, Aug. 2005.
[17] Y. B. Zhao, G. P. Liu, and D. Rees, "Integrated predictive control and scheduling co-design for networked control systems," IET Control Theory Appl., Vol. 2, pp. 7-15, Jan. 2008.

[18] F. Gil-Castineira, F. J. Gonzalez-Castano, and L. Franck, "Extending vehicular CAN fieldbuses with delay- tolerant networks," IEEE Trans. on Ind. Electron., Vol.55, No.9, pp. 3307-3314, Sep. 2008.

[19] W. Prodanov, M. Valle, and R. Buzas, "A Controller Area Network Bus Transceiver Behavioral Model for Network Design and Simulation," IEEE Trans. on Ind. Electron., Vol.56, No.9, pp.3762-3771, Sep. 2009.

[20] B. K. Bose, Modern Power Electronics and AC Drives, Prentice Hall, 2001.

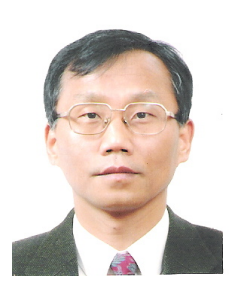

Tae-Won Chun was born in Korea in 1959. He received his B.S. in Electrical Engineering from Pusan National University, Korea, in 1981, and his M.S. and Ph.D. in Electrical Engineering from Seoul National University, Korea, in 1983 and 1987, respectively. Since 1986, he has been a member of the faculty in the Department of Electrical Engineering, Ulsan University, Korea, where he is currently a Full Professor. He was with the Department of Electrical and Computer Engineering, University of Tennessee, USA, as a Visiting Scholar from 1996 to 1997. From 2005 to 2006, he also served as a Visiting Scholar with the Department of Electrical and Computer Engineering, Virginia Polytechnic Institute and State University, USA. His current research interests include the control of electrical machines and power conditioning systems.

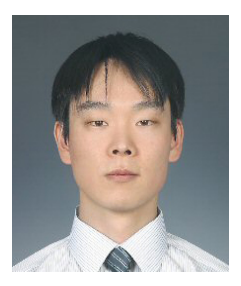

Jung-Ryol Ahn was born in Korea in 1976. He received his B.S. in Electrical and Electronics Engineering from Wonkyang University, Korea, in 2002, and his M.S. and $\mathrm{Ph} . \mathrm{D}$. in Electrical Engineering from Ulsan University, Korea, in 2004 and 2009, respectively. He is currently the Senior Researcher of the Future Automobile \& Components Research Center at DH Holdings Co. Ltd. Korea. His current research interest includes the control of electric vehicles.

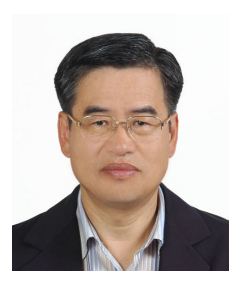

Hong-Hee Lee was born in Korea in 1957. He received his B.S., M.S. and Ph.D. in Electrical Engineering from Seoul National University, Korea, in 1980, 1982 and 1990, respectively. Since 1985, he has been a member of the faculty in the Department of Electrical Engineering, Ulsan University, Korea, where he is currently a Full Professor. He was with the Department of Electrical Engineering, University of Texas A\&M, USA as a Visiting Scholar from 1990 to 1991. His current research interests include wind power generation, matrix converters, and network-based factory automations.

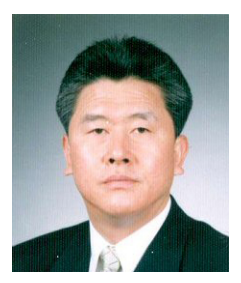

Heung-Geun Kim was born in Korea in 1956. He received his B.S., M.S. and Ph.D. in Electrical Engineering from Seoul National University, Korea, in 1980, 1982 and 1988, respectively. Since 1984, he has been a member of the faculty in the Department of Electrical Engineering, Kyungpook University, Korea, where he is currently a Full Professor. He was with the Department of Electrical Engineering, University of Wisconsin-Madison, USA, as a Visiting Scholar from 1990 to 1991. From 2006 to 2007, he also served as a Visiting Scholar with the Department of Electrical and Computer Engineering, Michigan State University, USA. His current research interests include the control of ac machines and PV power generation.

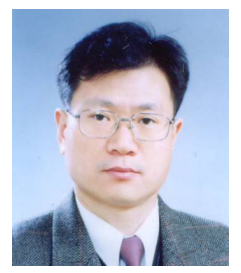

Eui-Cheol Nho was born in Korea in 1960. He received his B.S. in Electrical Engineering from Seoul National University, Korea, in 1984, and his M.S. and Ph.D. in Electrical and Electronics Engineering from KAIST, Korea, in 1986 and 1991, respectively. Since 1995, he has been a member of the faculty in the Department of Electrical Engineering, Pukyong University, Korea. He was with the Department of Electrical Engineering, University of Wisconsin-Madison, USA, as a Visiting Scholar from 1997 to 1998 . From 2005 to 2006, he also served as a Visiting Scholar with the Department of Electrical Engineering and Computer Science, University of California, Irvine, USA. His current research interests include the control of dynamo UPS, power line conditioners, etc. 